\title{
SARS-CoV-2: ¿está presente en la conjuntiva?
}

\section{SARS-CoV-2: is it present in the conjunctiva?}

Héctor J. Pérez-Cano*, Óscar Morales-López, Monserrat Y. Garrido-Santos y Selma A. Somilleda-Ventura

Centro de Investigación Biomédica, Fundación Hospital Nuestra Señora de la Luz I.A.P., Ciudad de México, México

\section{Resumen}

La familia Orthocoronavirinae fue descubierta en la década de 1960. Son virus envueltos de ARN monocatenario positivo y el tamaño de su genoma varía aproximadamente entre 26 y 32 kb, siendo el más grande para un virus ARN. En México, el primer caso reportado fue el de una persona proveniente de Italia. Tomando este como día 1, los casos se han ido incrementando y, hasta el momento de escribir el presente artículo, se tenían contabilizadas 133,974 personas infectadas y 15,944 decesos. La conjuntiva se ha propuesto como vía de entrada para el SARS-CoV-2. Sin embargo, en una búsqueda de material genético del coronavirus en muestras de lagrima y conjuntiva, los pacientes que no mostraban conjuntivitis resultaron negativos a la presencia de material genético viral, y solo una persona resultó positiva, tanto en muestras de lagrima como de conjuntiva. Los resultados negativos de ARN del SARS-CoV-2 en las lágrimas y las secreciones conjuntivales podría indicar que el virus no se replica en los epitelios conjuntivales, pero podría estar presente en cantidades no detectables, lo que no descarta que pueda ser transmitido a través de la conjuntiva.

Palabras clave: Conjuntiva. Coronavirus. Lágrima. Pandemia. SARS-CoV-2.

\section{Abstract}

The Orthocoronavirinae family was discovered in the 1960s. These are enveloped viruses with positive single-stranded RNA, and their genome size varies approximately between 26 and $32 \mathrm{~kb}$, being the largest genome for an RNA virus. In Mexico, the first reported case was that of a person from Italy. Taking this first case as day 1, the cases have been increasing and, at the time of writing this article, there were 133,974 infected people and 15,944 deaths. The conjunctiva has been proposed as a route of entry for SARS-CoV-2. However, in a search for coronavirus genetic material in tear and conjunctiva samples, patients who did not show conjunctivitis were negative for the presence of viral genetic material, and only one person was positive, both in tear and conjunctiva samples. Negative results for SARS-CoV-2 RNA in tears and conjunctival secretions could indicate that the virus does not replicate in conjunctival epithelia, but could be present in undetectable amounts, which does not rule out that it can be transmitted through the conjunctiva.

Keywords: Conjunctiva. Coronavirus. Tear. Pandemic. SARS-CoV-2.

\footnotetext{
Correspondencia:

*Héctor J. Pérez-Cano

Ezequiel Montes 135

Col. Tabacalera, Del. Cuauhtémoc

C.P. 06030, Ciudad de México, México Fecha de recepción: 12-06-2020

E-mail: drhectorpc @ hotmail.com; $\quad$ Fecha de aceptación: 15-04-2021

drhectorpc@gmail.com

DOI: 10.24875/RMO.M21000178

Disponible en internet: 02-06-2021 Rev Mex Oftalmol. 2021;95(5):209-214

www.rmo.com.mx 0187-4519/@ 2021 Sociedad Mexicana de Oftalmología. Publicado por Permanyer. Este es un artículo open access bajo la licencia CC BY-NC-ND (http://creativecommons.org/licenses/by-nc-nd/4.0/).
} 


\section{Introducción}

La familia Orthocoronavirinae, comúnmente conocida como coronavirus, fue descubierta en la década de 1960. Se trata de virus envueltos de ARN monocatenario positivo y su genoma varía aproximadamente entre 26 y $32 \mathrm{~kb}^{1}$. Infectan principalmente a animales vertebrados; su presencia se ha relacionado principalmente en murciélagos, aunque se han encontrado en ratones, felinos, bovinos y dromedarios ${ }^{2}$. Las mutaciones en el genoma viral han hecho posible que los coronavirus puedan transmitirse de los animales a los humanos, provocando un daño en la salud y originando la amenaza de brotes epidémicos ${ }^{3}$. Sus diferentes tipos provocan enfermedades respiratorias que pueden presentarse como un síndrome respiratorio leve, similar a un resfriado, hasta un síndrome respiratorio agudo grave $^{2,4}$. El coronavirus debe su nombre al aspecto que presenta, pues las microfotografías muestran una estructura con espículas que fueron descritas como una corona ${ }^{5}$. La enfermedad se propaga por contacto de persona a persona, a través de partículas que se expanden por el aire al hablar, toser o estornudar ${ }^{6}$.

\section{Método}

En este trabajo nos centramos en una revisión acerca del nuevo coronavirus que está afectando a todo el mundo, vinculado a un origen zoonótico y que presenta afectación ocular, principalmente conjuntivitis. Realizamos una breve descripción general del comportamiento de la pandemia en el mundo y en México, haciendo uso de la base de datos de la Universidad Johns Hopkins (disponible en https://coronavirus.jhu. edu/map.html) y de los datos nacionales del Gobierno Federal de México (disponibles en https://coronavirus. gob.mx/datos/), que constantemente actualiza la información. Se llevó a cabo una revisión de la literatura en las bases de datos PubMed y ScienceDirect. En PubMed, para la estrategia de búsqueda se usaron los operadores booleanos AND y OR de la siguiente manera: "SARS-CoV-2 OR Ocular manifestations AND conjunctiva". Se buscó también bibliografía en español en las bases de datos Medigraphic y Scielo. Los artículos elegidos se publicaron en su mayoría entre los años 2015 y 2020, aunque fueron necesarios algunos de más antigüedad para datos generales. Los temas estuvieron relacionados con la estructura del coronavirus, las fuentes de transmisión, los métodos diagnósticos, la epidemiología, el manejo de la COVID-19, las formas de prevención y el cuadro clínico.

\section{Resultados}

Se encontraron 257 artículos, de los cuales, al filtrarlos por título y contenido, y haciendo referencia a temas relacionados con coronavirus diferentes del SARS-CoV-2, solo utilizamos 34 (tres de ellos en español). Debido a la poca información existente hasta la fecha con respecto al tema, a continuación se describen los antecedentes y los conocimientos obtenidos de otros virus que ocasionaron brotes epidémicos y que pertenecen a la familia de los coronavirus, y se hace un breve resumen del comportamiento epidemiológico del SARS-CoV-2.

\section{Antecedentes}

En los últimos años se han presentado brotes epidémicos causados por coronavirus. En 2002 se describió una enfermedad respiratoria atípica, denominada SARS (Severe Acute Respiratory Syndrome), y en 2003 ya habían sido diagnosticados más de 8000 pacientes en 30 países, con más de 900 muertes (el promedio de letalidad reportado fue del $10 \%$ ). El virus identificado se denominó SARS-CoV ${ }^{4,7}$.

En el año 2012, en Arabia Saudita se detectó una enfermedad respiratoria que involucraba fiebre, tos, dificultad para respirar y neumonía, y que progresaba a insuficiencia respiratoria aguda, falla multiorgánica y muerte. Esta enfermedad era provocada por un nuevo coronavirus, identificado como nCoV, que posteriormente se renombró como MERS-CoV (Middle East Respiratory Syndrome Coronavirus) $)^{4,7}$.

En diciembre de 2019 surgió un nuevo coronavirus, llamado 2019-nCoV y posteriormente SARS-CoV-2 (Severe Acute Respiratory Syndrome Coronavirus 2). Su origen se reportó en la ciudad de Wuham, en Hubei, provincia de China ${ }^{8}$. Este virus se propagó rápidamente hasta ser una pandemia; a la fecha (agosto de 2020), se han reportado más de $23,700,000$ personas infectadas y más de 814,000 muertes en todo el mundo (https://coronavirus.jhu.edu/map.html).

\section{Casos en México}

En México, el primer caso reportado fue el de una persona proveniente de Italia. Tomando este como día 1 , los casos se han ido incrementando y, hasta el día 180 de la epidemia, se tenían contabilizadas 568,521 personas infectadas y 61,450 decesos; el índice de letalidad aumentó progresivamente hasta estabilizarse en un promedio del $10 \%$. El comportamiento de la 


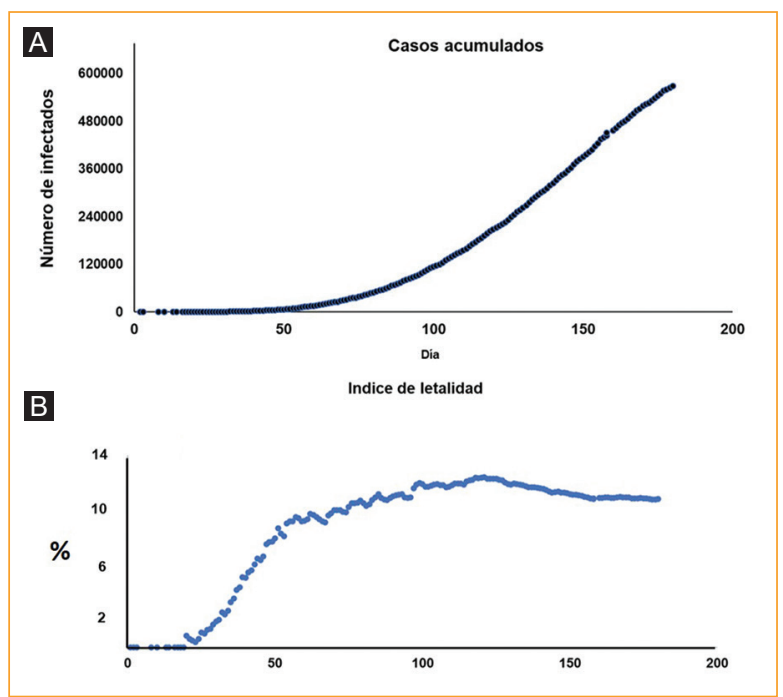

Figura 1. Comportamiento de la infección por SARS-CoV-2 hasta el día 180 de la llegada del virus a México, a partir del primer paciente detectado. A: casos acumulados por día (568,521 casos reportados). B: índice de letalidad. Datos obtenidos de https://coronavirus.gob.mx/.

infección sigue la tendencia que se muestra en la figura 1.

Se ha observado que la infección por SARS-CoV-2 es ligeramente mayor en los hombres (55-60\%) que en las mujeres ${ }^{5}$ (https://coronavirus.gob.mx/datos/). Se sabe que las personas infectadas con el SARS-CoV-2 pueden ser asintomáticas o presentar enfermedad de leve a grave, reportándose un $60 \%$ de pacientes ambulatorios y un $40 \%$ que han requerido hospitalización. Los signos y síntomas típicos se presentan en la tabla 19-12. Las complicaciones, como la neumonía y la insuficiencia de diversos órganos, ocurren principalmente en personas mayores y en las que presentan enfermedades crónicas como diabetes, hipertensión, afecciones cardiovasculares y otras que provocan inmunosupresión ${ }^{8,13,14}$. De manera interesante, se ha reportado que los niños muestran cierta resistencia a la enfermedad, pero representan un riesgo de diseminación viral, por lo que es importante tomarlo en cuenta para las medidas de contención de la pandemia ${ }^{15}$.

\section{Vías de transmisión}

Desde la aparición del SARS-CoV se han estudiado las vías de transmisión del virus, que se encuentra presente en las mucosas y las secreciones, y también se ha aislado de heces y orina ${ }^{16-19}$. Los casos de contagio han sido por contacto estrecho de persona a persona,
Tabla 1. Signos y síntomas que se presentan en la infección por SARS-CoV-2

\begin{tabular}{|l|c|}
\hline Signo o síntoma & Presentación en pacientes \\
\hline Fiebre & $88 \%$ \\
\hline Tos seca & $68 \%$ \\
\hline Fatiga & $38 \%$ \\
\hline Producción de esputo & $33 \%$ \\
\hline Dificultad para respirar & $19 \%$ \\
\hline Dolor de garganta & $14 \%$ \\
\hline Dolor de cabeza & $14 \%$ \\
\hline Mialgia o artralgia & $15 \%$ \\
\hline Escalofríos & $11 \%$ \\
\hline Náuseas o vómitos & $5 \%$ \\
\hline Congestión nasal & $5 \%$ \\
\hline Diarrea & $4 \%$ \\
\hline Hemoptisis & $0.9 \%$ \\
\hline Congestión conjuntival & $0.8 \%$ \\
\hline
\end{tabular}

definiendo el contacto estrecho como personas que cuidan o conviven con una persona enferma, y puede deberse a besar, abrazar, compartir utensilios de comida o bebida, o conversar a corta distancia, y también por una exploración médica ${ }^{20}$. Con respecto al SARS-CoV-2, se describe que se une a una proteína, la enzima convertidora de angiotensina 2, que se encuentra en la superficie de las células pulmonares, del corazón, los riñones y en el aparato gastrointestinal ${ }^{21,22}$. Se ha reportado que dicha enzima se expresa en el epitelio corneal, límbico y conjuntival, lo que explicaría por qué el ojo es una vía de entrada y la razón de que algunos pacientes presenten alteraciones oculares durante el cuadro clínico ${ }^{23,24}$. Las lágrimas se han considerado un fluido corporal que puede albergar potencialmente el coronavirus, pero es una cuestión que continúa en discusión puesto que los estudios no han sido concluyentes con respecto a la presencia del virus en las lágrimas o la conjuntiva ${ }^{20,25-27}$.

Por otro lado, la superficie ocular se ha estimado en 1600 a $1869 \mathrm{~mm}^{2}$ en cada ojo, incluida la córnea, lo que representa un área de absorción máxima de $3738 \mathrm{~mm}^{2}$ aproximadamente, que incluye la conjuntiva tarsal y los fondos palpebrales. Sabemos que la producción de lágrima permite al ojo evitar la intrusión de material extraño, entre ellos los microorganismos, y 
además se ha demostrado que las pestañas desvían los flujos de aire y así controlan la entrada de partículas. La deficiencia en estos mecanismos podría aumentar el riesgo de infección ${ }^{28}$.

\section{Coronavirus en la conjuntiva}

Desde el inicio de la pandemia por el nuevo virus SARS-CoV-2, la oftalmología ha desempeñado un papel importante en la vigilancia del comportamiento de la enfermedad. Recordemos que el Dr. Li Wenliang, oftalmólogo del Hospital Central de Wuham, advirtió sobre el posible brote de la enfermedad parecida al SARS después de atender a pacientes con afectación oftalmológica y síntomas de falla respiratoria ${ }^{28}$. La identificación del uso de la enzima convertidora de la angiotensina 2 por los coronavirus ha proporcionado información sobre la permisividad del tejido ocular a la infección por estos virus; sin embargo, aunque la información para el SARS-CoV-2 aún es escasa, podemos ver que el comportamiento de los virus pertenecientes a esta familia es similar ${ }^{21}$.

Para tratar de responder a la pregunta de si el SARSCoV-2 se encuentra en la conjuntiva podemos revisar lo que se conoce acerca de otros virus; por ejemplo, al final de la crisis del SARS-CoV se identificó un nuevo coronavirus humano, el HCoV-NL63, que fue aislado de un niño de 7 meses que presentó bronquiolitis y conjuntivitis ${ }^{29}$. Vabret, et al. ${ }^{30}$ reportaron que el $17 \%$ de los pacientes con HCoV-NL63 desarrollaron conjuntivitis, pero a la fecha no hay estudios que detallen los mecanismos de infección en tejidos oculares ${ }^{31}$. Chan, et al. ${ }^{25}$ reportan que 5 de 17 pacientes fueron positivos para SARS-CoV utilizando la técnica de reacción en cadena de la polimerasa con transcripción reversa (RT-PCR) en muestras de nasofaringe, pero en las muestras de raspado conjuntival el virus no pudo ser detectado ni aislado en cultivo.

Con respecto al SARS-CoV-2, Wu, et al. ${ }^{27}$ estudiaron 38 pacientes que, además de síntomas respiratorios, presentaban epifora, congestión conjuntival y quemosis. Se determinó la presencia de material genético viral por RT-PCR y se encontró una positividad del $91 \%$ en muestras de raspado nasofaríngeo, mientras que solo el $16.7 \%$ de las muestras de raspado conjuntival fueron positivas.

$\mathrm{Xia}$, et al. ${ }^{29}$ realizaron una búsqueda de material genético del SARS-CoV-2 utilizando RT-PCR en muestras de lágrima y conjuntiva de 30 pacientes, y obtuvieron dos muestras en tiempos diferentes (intervalo de 2 a 3 días). Veintinueve de estos pacientes no mostraban conjuntivitis y resultaron negativos a la presencia de material genético viral; solo un paciente resultó positivo para SARS-CoV-2 en muestras tanto de lágrima como de conjuntiva.

Deng, et al. ${ }^{32}$ han reportado datos de un modelo animal que consistió en la inoculación de SARS-CoV-2 en la conjuntiva de monos rhesus (Macaca mulatta), y solo detectaron SARS-CoV-2 en hisopos conjuntivales el primer día después de la inoculación, mientras que en los hisopos nasales y faríngeos la presencia del virus se detectó hasta 7 días después de la inoculación. Los resultados sugieren que la probabilidad de infección por el SARS-CoV-2 es más alta por vía de la nariz y la garganta que por la conjuntiva. Sin embargo, el virus podría ser transportado por las lágrimas a través del conducto lagrimal para causar la infección del tracto respiratorio.

\section{Discusión}

Se ha observado que las personas infectadas por algún tipo de coronavirus pueden presentar alguna manifestación ocular, principalmente conjuntivitis, lo que sugiere que el virus se podría localizar en esta parte anatómica y constituiría un riesgo de transmisión; sin embargo, los estudios realizados en busca de material genético viral han tenido resultados positivos bajos, que van del $0 \%$ al $16.7 \%$ de positividad ${ }^{29-32}$. Se ha especulado que la absorción viral en el tejido ocular es más rápida que en otros tejidos, por lo que la presencia del virus en la conjuntiva podría durar un lapso corto de tiempo pero causar la respuesta inflamatoria y las manifestaciones oculares que son más duraderas. La hipótesis más aceptada hasta ahora es que la vía conjuntival no es la preferida por el SARS-CoV-2 y que el drenaje lagrimal podría estar implicado en la baja adherencia de las partículas virales al epitelio conjuntival, realizando un efecto de «lavado» y llevando al virus al conducto lagrimal para posteriormente ingresar al sistema respiratorio 28,32 . A pesar de que estos datos sugieren que el riesgo de transmisión del SARS-CoV-2 a través de la conjuntiva es muy bajo, existe y debe ser tomado en cuenta. Se ha observado que los médicos oftalmólogos tienen un alto riesgo de infección, no por transmisión por presencia del virus en la conjuntiva del paciente, sino por el contacto cercano debido a la distancia tan corta que existe en la exploración oftalmológica, por lo que se debe tener en cuenta el uso de un equipo de protección adecuado para la atención oftalmológica ${ }^{33}$. 
Los resultados negativos de ARN del SARS-CoV-2 en las lágrimas y las secreciones conjuntivales podrían indicar que el virus no se replica en los epitelios conjuntivales, pero podría estar presente en cantidades no detectables, lo que no descarta que pueda ser transmitido a través de la conjuntiva ${ }^{29-31}$. Las limitantes de los reportes descritos es que los estudios se han realizado con tamaños de muestra pequeños y no excluyen que se trate de resultados falsos negativos; si la muestra fuera más amplia, la sensibilidad podría aumentar, y por otro lado es importante descartar que el virus y su material genético solo se encuentren presentes durante un corto tiempo, por lo que es necesario realizar una investigación que permita corroborar esta hipótesis. Otro aspecto importante es verificar tanto la sensibilidad como la especificidad de las pruebas para detectar el ARN viral, así como los sistemas de transporte de la muestra, pues no hay que olvidar que el ARN es un material muy lábil y esto podría estar contribuyendo a los falsos negativos ${ }^{34}$.

\section{Conclusiones}

La vía de entrada del SARS-CoV-2 es principalmente a través de los ojos, la nariz y la garganta. Sin embargo, los datos sugieren que no se establece la infección del epitelio conjuntival, sino que el virus es transportado a la vía nasal a través del conducto lagrimal. Es importante considerar que la conjuntivitis puede ser una presentación de la enfermedad por SARS-CoV-2 y que, a pesar de los hallazgos negativos de su presencia en la conjuntiva, no se debe ignorar la probable transmisión a través de la superficie ocular.

¿El coronavirus está presente en la conjuntiva? Podría ser que, como vía de entrada, su presencia sea momentánea y pase al sistema respiratorio a través del conducto lagrimal, pero la pregunta no está del todo respondida y es necesario realizar una mayor investigación.

\section{Financiamiento}

La presente investigación no ha recibido ayudas específicas provenientes de agencias del sector público, sector comercial ni entidades sin ánimo de lucro.

\section{Conflicto de intereses}

Los autores no tienen ningún conflicto de intereses que revelar con respecto a esta revisión.

\section{Responsabilidades éticas}

Protección de personas y animales. Los autores declaran que para esta investigación no se han realizado experimentos en seres humanos ni en animales.

Confidencialidad de los datos. Los autores declaran que en este artículo no aparecen datos de pacientes.

Derecho a la privacidad y consentimiento informado. Los autores declaran que en este artículo no aparecen datos de pacientes.

\section{Bibliografía}

1. Cui J, Li F, Shi ZL. Origin and evolution of pathogenic coronaviruses. Nat Rev Microbiol. 2019;17:181-92.

2. Hu B, Ge X, Wang LF, Shi Z. Bat origin of human coronaviruses. Virol J. 2015;12:221.

3. Siddell SG, Anderson R, Cavanagh D, Fujiwara K, Klenk HD, Macnaughton MR, et al. Coronaviridae. Intervirology. 1983;20:181-9.

4. de Wit E, van Doremalen N, Falzarano D, Munster VJ. SARS and MERS: recent insights into emerging coronaviruses. Nat Rev Microbiol. 2016; 14:523-34.

5. Gorbalenya AE, Snijder EJ, Spaan WJM. Severe acute respiratory syndrome coronavirus phylogeny: toward consensus. J Virol. 2004;78:7863-6.

6. Oxford JS, Bossuyt S, Lambkin R. A new infectious disease challenge: Urbani severe acute respiratory syndrome (SARS) associated coronavirus. Immunology. 2003;109:326-8.

7. Mackay IM, Arden KE. MERS coronavirus: diagnostics, epidemiology and transmission. Virol J. 2015;12:222.

8. Mo P, Xing Y, Xiao Y, Deng L, Zhao Q, Wang H, et al. Clinical characteristics of refractory COVID-19 pneumonia in Wuhan, China. Clin Infect Dis. 2020. Mar 16:ciaa270. doi: 10.1093/cid/ciaa270. Online ahead of print.

9. Peng Y, Zhou YH. Is novel coronavirus disease (COVID-19) transmitted through conjunctiva? J Med Virol. 2020;92:1408-9.

10. Jin X, Lian JS, Hu JH, Gao J, Zheng L, Zhang YM, et al. Epidemiological, clinical and virological characteristics of 74 cases of coronavirus-infected disease 2019 (COVID-19) with gastrointestinal symptoms. Gut. 2020; 69:1002-9.

11. Kannan S, Shaik Syed Ali P, Sheeza A, Hemalatha K. COVID-19 (novel coronavirus 2019) - recent trends. Eur Rev Med Pharmacol Sci. 2020;24:2006-11.

12. Madabhavi I, Sarkar M, Kadakol N. COVID-19: a review. Monaldi Arch Chest Dis. 2020;90(2)

13. Devaux CA, Rolain JM, Raoult D. ACE2 receptor polymorphism: susceptibility to SARS-CoV-2, hypertension, multi-organ failure, and COVID-19 disease outcome. J Microbiol Immunol Infect. 2020:53:425-35.

14. Atluri S, Manchikanti L, Hirsch JA. Expanded umbilical cord mesenchymal stem cells (UC-MSCs) as a therapeutic strategy in managing critically ill COVID-19 patients: the case for compassionate use. Pain Physician. 2020;23:E71-83.

15. Cheng ZJ, Shan J. 2019 Novel coronavirus: where we are and what we know. Infection. 2020;48:155-63.

16. Peng $X, X u X$, Li $Y$, Cheng $L$, Zhou $X$, Ren B. Transmission routes of 2019-nCoV and controls in dental practice. Int J Oral Sci. 2020;12:9.

17. Ge ZY, Yang LM, Xia JJ, Fu XH, Zhang YZ. Possible aerosol transmission of COVID-19 and special precautions in dentistry. J Zhejiang Univ Sci B. 2020;21:361-8.

18. Setti L, Passarini F, De Gennaro G, Barbieri P, Perrone MG, Borelli M, et al. Airborne transmission route of COVID-19: why 2 meters $/ 6$ feet of inter-personal distance could not be enough. Int J Environ Res Public Health. 2020;17:2932.

19. Chen Y, Chen L, Deng Q, Zhang G, Wu K, Ni L, et al. The presence of SARS-CoV-2 RNA in the feces of COVID-19 patients. J Med Virol. 2020;92:833-40

20. Hernández G. SARS: epidemiología y mecanismos de transmisión. Med Intensiva 2003:27:686-91.

21. Cano F, Gajardo M, Freundlich M. Eje renina angiotensina, enzima convertidora de angiotensina 2 y coronavirus. Rev Chil Pediatr. 2020:91:330-8.

22. Soler MJ, Lloveras J, Batlle D. Enzima conversiva de la angiotensina 2 y su papel emergente en la regulación del sistema renina-angiotensina. Med Clin (Barc). 2008;131:230-6. 
Rev Mex Oftalmol. 2021;95(5)

23. Zhou L, Xu Z, Castiglione GM, Soiberman US, Eberhart CG, Duh EJ. ACE2 and TMPRSS2 are expressed on the human ocular surface, suggesting susceptibility to SARS-CoV-2 infection. Ocul Surf. 2020;18:537-44.

24. Collin J, Queen R, Zerti D, Dorgau B, Georgiou M, Djidrovski I, et al. Co-expression of SARS-CoV-2 entry genes in the superficial adult human conjunctival, limbal and corneal epithelium suggests an additional route of entry via the ocular surface. Ocul Surf. 2021;19:190-200.

25. Chan WM, Yuen KS, Fan DS, Lam DS, Chan PK, Sung JJ. Tears and conjunctival scrapings for coronavirus in patients with SARS. Br J Ophthalmol. 2004;88:968-9.

26. Seah I, Agrawal R. Can the coronavirus disease 2019 (COVID-19) affect the eyes? A review of coronaviruses and ocular implications in humans and animals. Ocul Immunol Inflamm. 2020;16:1-5.

27. Wu P, Duan F, Luo C, Liu Q, Qu X, Liang L, et al. Characteristics of ocular findings of patients with coronavirus disease 2019 (COVID-19) in Hubei Province, China. JAMA Ophthalmol. 2020;138:575-8.

28. Coroneo MT. The eye as the discrete but defensible portal of coronavirus infection. Ocul Surf. 2021;19:176-82.
29. Xia J, Tong J, Liu M, Shen Y, Guo D. Evaluation of coronavirus in tears and conjunctival secretions of patients with SARS-CoV-2 infection. J Med Virol. 2020;92:589-94.

30. Vabret A, Mourez T, Dina J, van der Hoek L, Gouarin S, Petitjean J, et al. Human coronavirus NL63, France. Emerg Infect Dis. 2005;11:1225-9.

31. Durán CSC, Mayorga GDC. The eye: "An organ that must not be forgotten in coronavirus disease 2019 (COVID-2019) pandemic". J Optom. 2020;14:114-9.

32. Deng W, Bao L, Gao H, Xiang Z, Qu Y, Song Z, et al. Rhesus macaques can be effectively infected with SARS-CoV-2 via ocular conjunctival route. bioRxiv. 2020. Disponible en: https://www.biorxiv.org/content/10.1101/2020.03.13.990036v1

33. Li JO, Lam DSC, Chen Y, Ting DSW. Novel coronavirus disease 2019 (COVID-19): the importance of recognising possible early ocular manifestation and using protective eyewear. $\mathrm{Br} \mathrm{J}$ Ophthalmol. 2020;104:297-8.

34. Scohy A, Anantharajah A, Bodéus M, Kabamba-Mukadi B, Verroken A, Rodriguez-Villalobos $\mathrm{H}$. Low performance of rapid antigen detection test as frontline testing for COVID-19 diagnosis. J Clin Virol. 2020;129:104455. 